\title{
Is virtual reality beneficial for dual-task gait training in patients with Parkinson's disease?
}

\author{
A systematic review
}

\author{
Fernanda Freitag ${ }^{1,2}$, Sonia Maria Dozzi Brucki1 ${ }^{10}$, Alessandra Ferreira Barbosa ${ }^{2,3}$, \\ Janini Chen ${ }^{1,2}$, Carolina de Oliveira Souza ${ }^{1,2}$, Débora Francato Valente², \\ Hsin Fen Chien ${ }^{1,2}$, Cynthia Bedeschi4 ${ }^{4}$ Mariana Callil Voos ${ }^{1,2,3}$
}

\begin{abstract}
This systematic review examined the evidence about the effects of virtual reality (VR) on dual-task gait training in Parkinson's disease (PD). Methods: this study (PROSPERO registration CRD42019114736) aimed to answer the question: "Is VR beneficial for dual-task gait training in patients with PD?" We searched for studies from 2008 to 2018 on Medline/PubMed and Web of Science/Web of knowledge databases. The keywords were Parkinson AND gait training AND virtual reality OR Parkinson AND gait training AND game. A total of 55 articles were retrieved, of which 11 systematic reviews, 11 opinions, letters to the editor, posters or conferences abstracts and 17 studies not evaluating the effects of VR gait training were excluded. Three further studies addressing VR dual-task gait training in PD (found in references of studies selected) were also included. Therefore, 19 studies were included and analysed. Results: all studies reported gait improvement after VR training. Many clinical scales were used, hampering comparison of the effects of each protocol. Conclusion: VR dual-task gait training should be part of rehabilitation protocols for PD. The studies showed that VR training was effective, although specific guidelines have not yet been established.
\end{abstract}

Key words: Parkinson's disease, gait, cognition, virtual reality, videogames.

\section{A REALIDADE VIRTUAL É BENÉFICA PARA 0 TREINO DE MARCHA COM TAREFA DUPLA EM PACIENTES COM DOENÇA DE PARKINSON? REVISÃO SISTEMÁTICA}

RESUMO. Essa revisão sistemática examinou as evidências sobre os efeitos da realidade virtual (RV) no treinamento de marcha com tarefa-dupla na doença de Parkinson (DP). Métodos: esse estudo (registro PROSPERO: CRD42019114736) visou responder à questão: "A RV é benéfica no treinamento de marcha com tarefa-dupla em pacientes com DP?" A busca, de 2008 a 2018, foi feita nos bancos de dados Medline/PubMed e Web of Science/Web of Knowledge. Os descritores foram Parkinson, treinamento de marcha, realidade virtual e jogos eletrônicos. Foram encontrados 55 artigos e eliminadas 11 revisões sistemáticas, 11 cartas ao editor, resumos de pôsteres ou conferências e 17 estudos que não avaliaram os efeitos do treinamento da marcha em RV. Três outros estudos (encontrados nas referências dos estudos selecionados) foram incluídos porque também abordaram o treinamento de marcha com dupla tarefa de RV em DP. Portanto, 19 estudos foram incluídos e analisados. Resultados: todos os estudos relataram melhora da marcha após 0 treinamento com RV. Muitas escalas clínicas foram usadas e foi difícil comparar os efeitos de cada protocolo. Conclusão: RV deve fazer parte dos protocolos de reabilitação em DP. 0 treinamento com RV é eficaz, embora recomendações específicas ainda não tenham sido estabelecidas.

Palavras-chave: doença de Parkinson, marcha, cognição, realidade virtual, videogames.

This study was conducted at the Postgraduate program in Neurology, Department of Neurology, FMUSP, São Paulo, SP, Brazil.

'Postgraduate Program in Neurology, Department of Neurology, FMUSP, São Paulo, SP, Brazil. 2Rehabilitation in Movement Disorders (REMOVE) Research Group, São Paulo, SP, Brazil. 3Postgraduate Program in Rehabilitation Sciences. Physiotherapy, Speech Therapy and Occupational Therapy, FMUSP, São Paulo, SP, Brazil. 4Postgraduate program in Neuroscience and Behavior, Institute of Psychology, USP, São Paulo, SP, Brazil.

Mariana Callil Voos. Depto de Fisioterapia, Fonoaudiologia e Terapia Ocupacional / Faculdade de Medicina / Universidade de São Paulo - Rua Cipotânea, 51 05360-160 São Paulo SP - Brazil. E-mail: marivoos@usp.br

Disclosure: The authors report no conflicts of interest.

Received December 29, 2018. Accepted in final form April 09, 2019. 
P arkinson's disease (PD) is the second most common neurodegenerative disease, characterized by motor and cognitive symptoms. Executive dysfunction can be present from the early stages of PD. These deficits increase falls risk and reduce functional independence, especially in dual-task performance. Executive function has been defined as a group of abilities involved in solving problems, reaching goals and meeting environmental demands, ${ }^{1,2}$ such as cognitive flexibility and decision-making. ${ }^{3}$ More than $50 \%$ of patients with PD have frontal lobe dysfunction, which compromises attention, executive function, spatial perception and implicit/episodic memory. ${ }^{4}$

Patients with PD have a poorer standing balance than healthy individuals. ${ }^{5,6}$ While performing a secondary task associated with ambulation, patients with PD have lower gait speed, shorter step length and freezing of gait episodes. Postural control deficits result in decreased mobility and reduced functional independence, ${ }^{6}$ e.g. during the sit-to-stand-to-sit sequence, especially when under the dual-task condition. Therefore, patients with PD must deal with the disruption of their motor and cognitive performance when tackling the cognitive-motor demands of their tasks of daily living. ${ }^{6,7}$

Many studies have shown the importance of visual, auditory, verbal and mnemonic cues (e.g. mental practice). Although cues help attention engagement during balance and gait tasks ${ }^{2,4,8}$ they may compete with other cognitive components required in daily life dual- or multiple-tasks. For example, while crossing a street, the patient may evoke visual or auditory cues to maintain step length or cadence. However, this strategy may compete with the intrinsic visual and auditory stimuli of traffic lights, pedestrians and vehicles.

Dual-task training improves cognitive-motor performance and directs attentional focus on specific outcomes. This is important because of the similarity with everyday tasks, when people focus on outcomes rather than on maintaining balance. ${ }^{9}$ Virtual reality (VR) provides dual-task training and requires information processing, attentional shifting, sensory integration, motor planning, while VR may also provide feedback to enhance motor learning., ${ }^{9,10}$

Postural control requires the integration of visual, somatosensory and vestibular systems. Executive function contributes in adapting inputs to meet environmental demands. In older adults and patients with PD, the reduced speed of sensory processing, motor planning and muscle activation results in increased attentional demands to maintain stability. ${ }^{11}$ VR training may optimise all these components and, therefore, motor learning. It may also be safer and more motivating than conventional approaches. ${ }^{11-13}$ Thus, VR training may improve adherence by offering personalised and fun exercises, with progressive cognitive overload. ${ }^{14}$

VR provides complex environments for balance and gait training of patients with PD. Improvements in step and stride length, gait velocity, functional independence, quality of life, and cognitive function have been reported after VR interventions with PD patients. ${ }^{14,15}$. Therefore, VR can play an important role in motor control and learning in PD. ${ }^{16}$ Several VR dual-task gait training protocols have been proposed for patients with $\mathrm{PD}$, but the effects are not clear. This systematic review aimed to examine and analyse the evidence on VR dualtask gait training in PD.

\section{METHODS}

This systematic review followed the Preferred Reporting Items for Systematic Reviews and Meta-Analyses (PRISMA) checklist. The study was registered on the International Prospective Register of Systematic Reviews (PROSPERO) under registration number CRD42019114736. Two researchers conducted the data search independently and blindly. Another three researchers conducted the data review and all researchers discussed all cases of doubt. The study aimed to answer the question: "Is VR beneficial for dual-task gait training in patients with $\mathrm{PD}$ ?"

The inclusion criteria were studies available on Medline/ Pubmed and Web of Science/ Web of Knowledge databases, which addressed VR dual-task gait training. The exclusion criteria were: studies that focused on drugs or surgery instead of dual-task training, literature reviews, abstracts and letters to the editor.

We searched studies published from 2008 to 2018 on Medline/ Pubmed and Web of Science/ Web of Knowledge databases. The keywords were Parkinson AND gait training AND virtual reality OR Parkinson AND gait training AND game.

The search was performed on August 26 ${ }^{\text {th }}, 2018$. A total of 55 articles were retrieved, of which 11 systematic reviews, 17 studies not specifically evaluating the effects of VR dual-task gait training and 11 opinions, letters to the editor or conference or poster abstracts were subsequently excluded. The references of these sixteen studies were also analysed. Three further studies focusing on VR dual-task gait training in PD were also included. Therefore, 19 studies were included in the qualitative synthesis (Figure 1). Figure 1 shows all the steps of the systematic review and the reasons for study exclusions. 


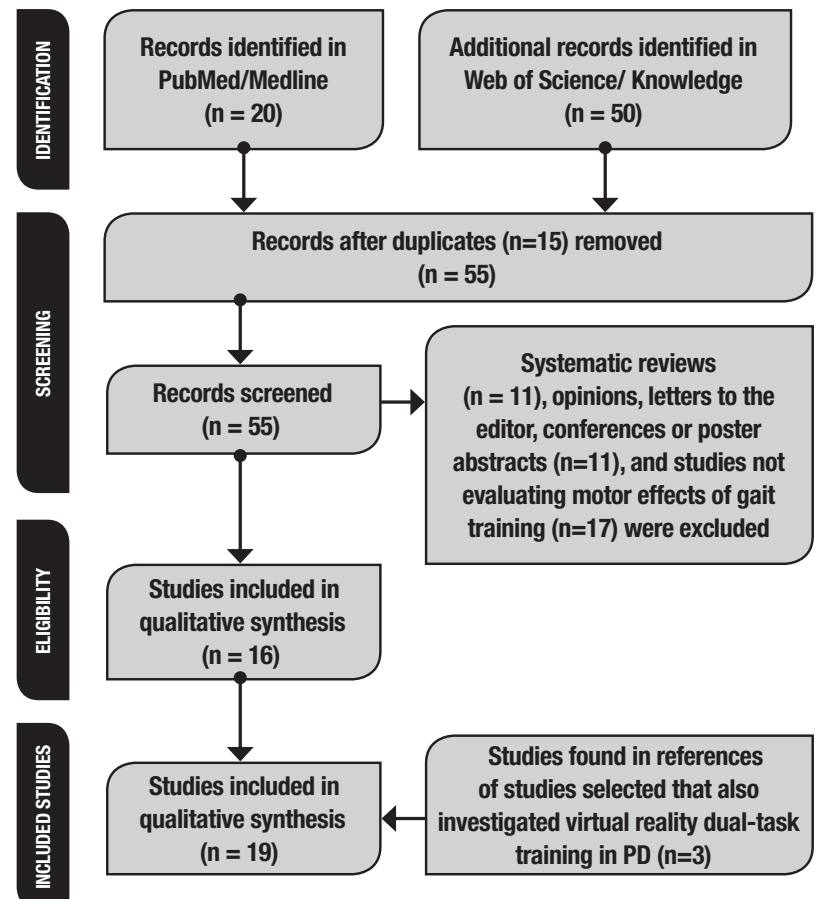

Figure 1. Studies included in systematic review, based on PRISMA criteria (2009): identification, screening, eligibility and studies included in review $(n=19)$.

The studies were also scored with the Physiotherapy Evidence Database (PEDro). This database evaluates the quality of each study and the validity of their conclusions based on the Delphi list (Verhagen et al., 1998). ${ }^{17}$ This scale has 11 domains, which evaluate: 1 . Eligibility criteria; 2. Random distribution of subjects in each group; 3 . Secret allocation of subjects; 4 . Similar groups regarding the most important prognosis; 5 . Blind participation of subjects; 6 . Blind participation of therapists; 7. Blind examiners; 8. At least one key result obtained in more than $85 \%$ of subjects; 9 . Subjects received treatment or control condition; 10. Intergroup statistical comparisons have been performed for at least one key outcome; 11 . Presence of precision and variability measures.

\section{RESULTS}

The 19 studies selected are summarized in Table 1. Several clinical scales were used, but the most frequent were Parkinson's Disease Questionnaire-39, Unified Parkinson Disease Rating Scale-III, Dynamic Gait Index and the 10-Minute Walk Test. Most studies described gait improvement after virtual reality training. The sixminute walk test, the Montreal Canadian Cognitive Assessment, the timed up-and-go test and the Berg Balance Scale were also used in evaluation protocols, but less frequently.
Table 2 shows the score on each domain of the PEDro database. The scores on PEDro ranged from 4 to 11 , but 11 studies were scored as 8 or higher. Therefore, although protocols were variable, many studies showed high quality of evidence that supported VR dual-task gait training.

\section{DISCUSSION}

The present study investigated whether VR dual-task gait training would promote gait improvement in $\mathrm{PD}$ patients. Patients with PD experience loss of functional independence and quality of life and difficulties in activities of daily living. VR can increase motivation in rehabilitation programs, and improve gait, as observed in the nineteen studies from 2008 to 2018 included in the present review. Although the scores on the PEDro scale ranged from 4 to 11,11 studies were scored as 8 or higher. Therefore, many studies showed high quality of evidence that supported VR dual-task gait training.

VR opens a wide range of possibilities of therapeutic approaches, and involves several types of stimuli (sensory, motor, cognitive, psychological). Thus, VR training provides several dual-task demands, characterizing one of the training premises. Therefore, VR may optimize gait training through posture and balance improvement and cognitive training. ${ }^{20,23,37}$ Most VR tasks are cognitive-motor, such as activities of daily living., ${ }^{6,7}$

Many studies included in the present review showed improvements in speed, step length and cadence of gait, measured by kinematic analysis and/ or clinical scales, such as the Dynamic Gait Index, Timed Up-and-Go, 6-minute walking test or 10-meter walking test (Espay et al., 2010; ${ }^{18}$ Mirelman et al., 2011; $;^{19}$ Esculier et al., 2012;: ${ }^{20}$ Mhatre et al., 2013; ${ }^{21}$ Pompeu et al., 2014; ${ }^{22}$ Liao et al., 2015; ${ }^{25}$ Palacios-Navarro et al., 2015; ${ }^{27}$ Ginis et al., 2016;28 Yang et al., 2016; ${ }^{29}$ Strouwen et al., 2017; ${ }^{30}$ Gandolfi et al., 2017; ${ }^{31}$ Ferraz et al., 2018; ${ }^{32}$ de Melo et al., 2018; ${ }^{34}$ Alves et al., 2018 $8^{35}$ ). The improvement in gait speed may be attributed to higher motivation, or to the higher intensity (higher number of repetitions and/ or longer periods of training) promoted by the VR dualtask training. These training quality characteristics may also explain the more efficient cardiovascular adjustments, e.g. heart rate (de Melo et al., 2018 ${ }^{34}$ ).

Some authors reported improvement on static postural balance, as measured by the Berg Balance Scale, after VR dual-task gait training (Mhatre et al., 2013; ${ }^{21}$ Pompeu et al., 2014; ${ }^{22}$ Yang et al., 2015; ${ }^{29}$ Gandolfi et al., 2017 ${ }^{31}$ ). These protocols involved weight shifting and functional reach tasks, highly recommended in PD rehabilitation because they improve static and dynamic 


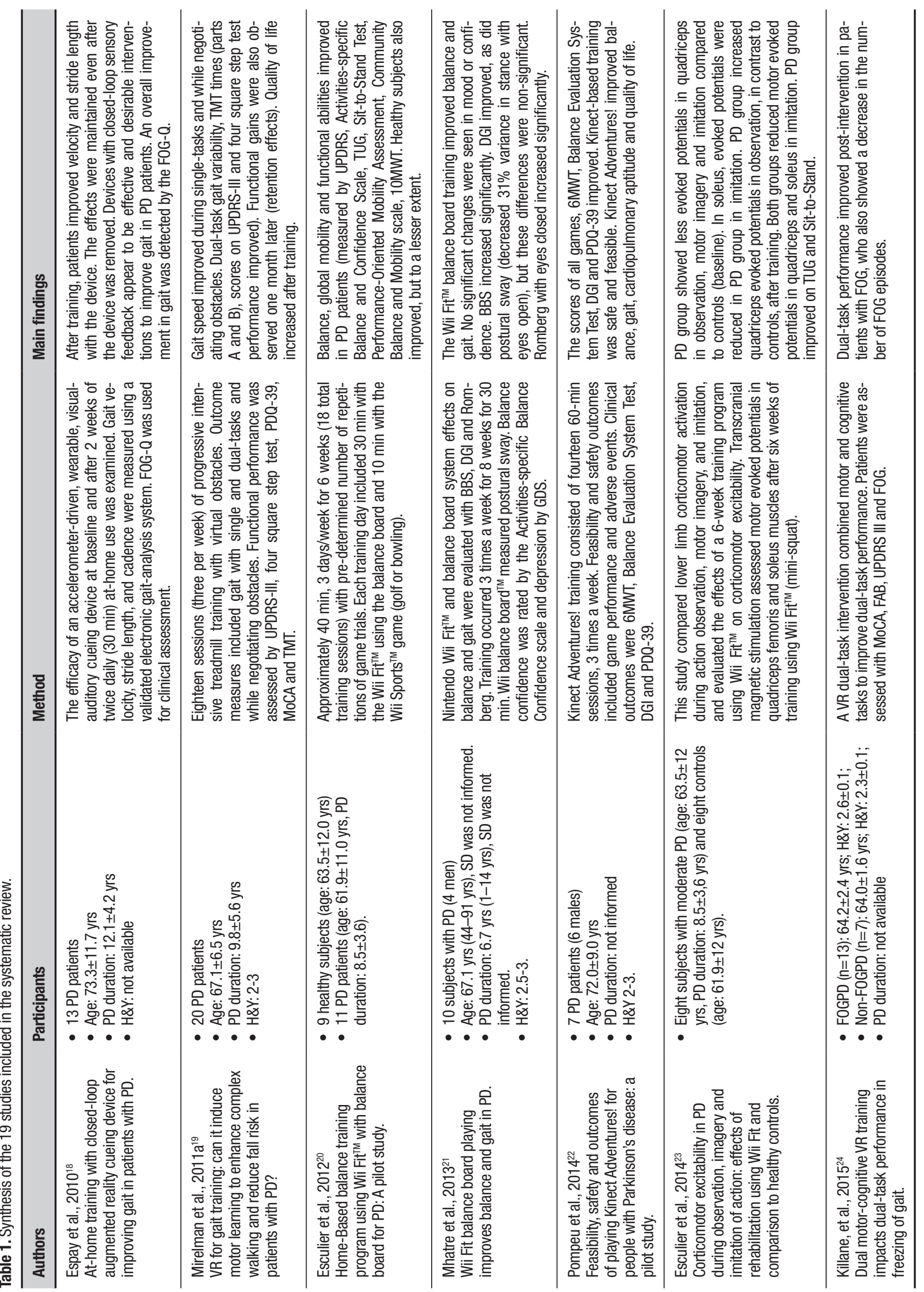




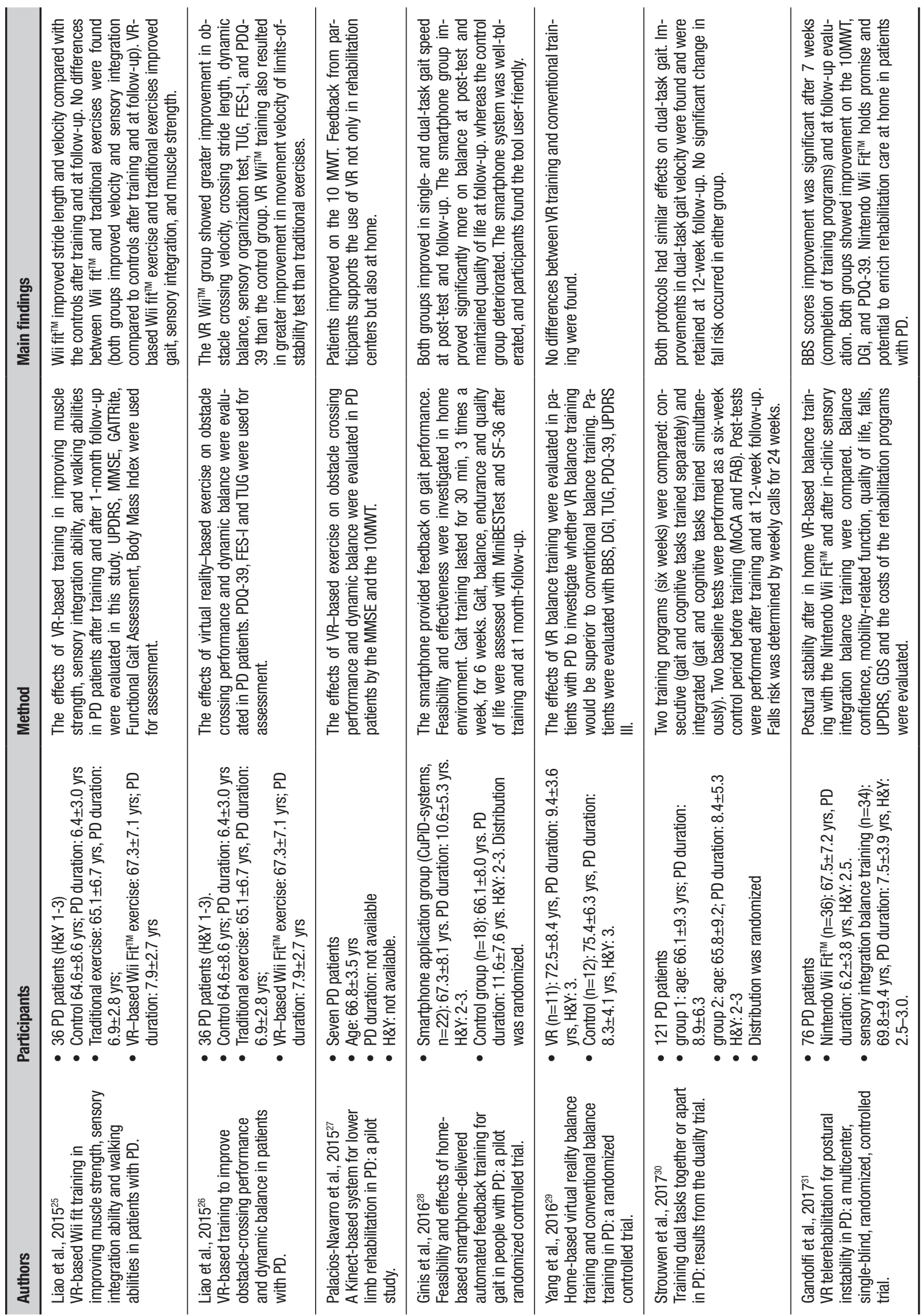




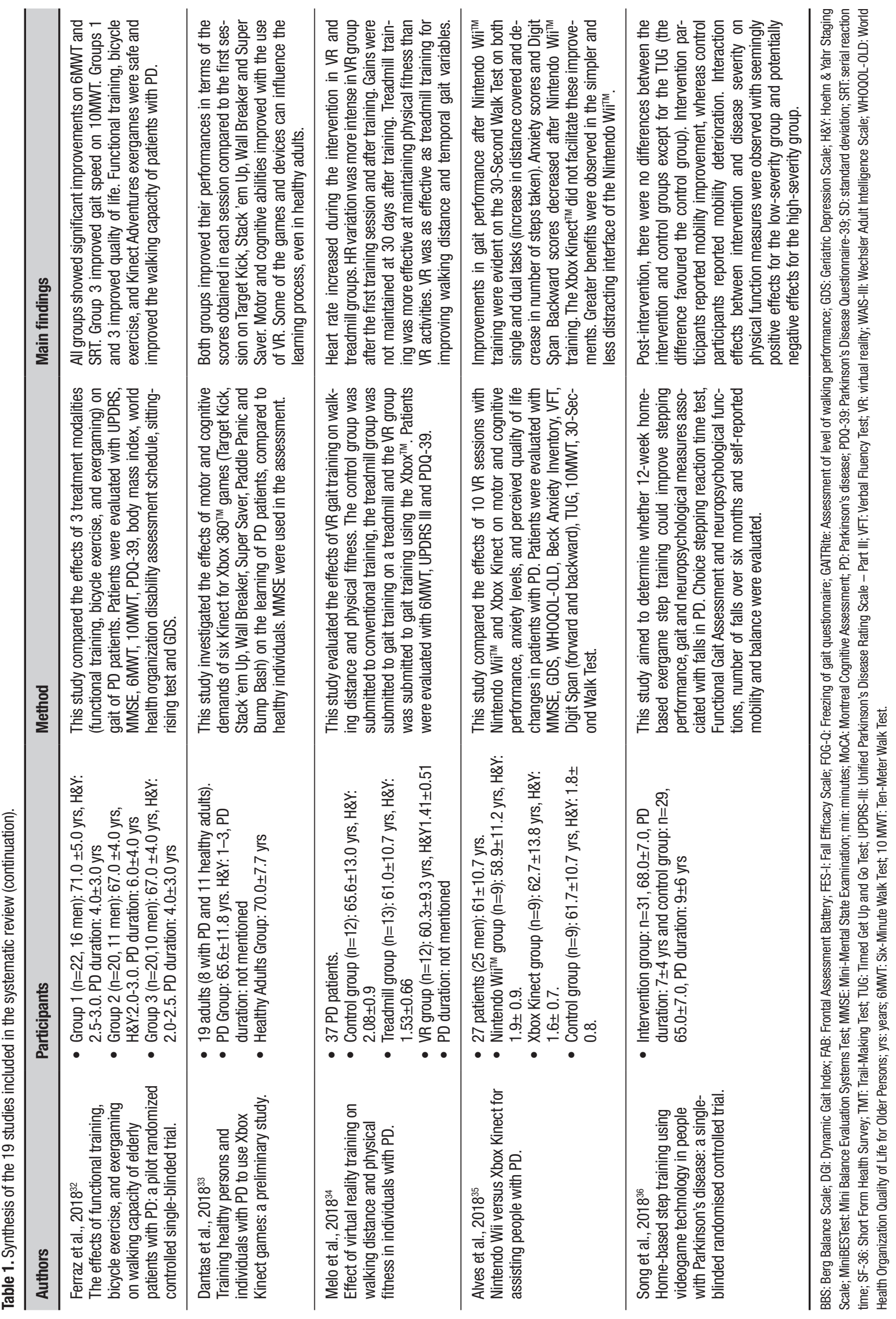


Table 2. PEDro database classification.

\begin{tabular}{|c|c|c|c|c|c|c|c|c|c|c|c|c|}
\hline Authors & 1 & 2 & 3 & 4 & 5 & 6 & 7 & 8 & 9 & 10 & 11 & Total \\
\hline Espay et al., $2010^{18}$ & Yes & Yes & No & Yes & No & No & No & Yes & Yes & Yes & Yes & 7 \\
\hline Mirelman et al., $2011^{19}$ & Yes & No & No & No & No & No & No & Yes & Yes & No & Yes & 4 \\
\hline Esculier et al., $2012^{20}$ & Yes & No & No & No & No & No & No & Yes & Yes & No & Yes & 4 \\
\hline Mhatre et al., $2013^{21}$ & Yes & Yes & No & Yes & No & No & No & Yes & Yes & Yes & Yes & 7 \\
\hline Esculier et al., $2014^{22}$ & Yes & No & No & Yes & No & No & No & Yes & Yes & No & Yes & 5 \\
\hline Pompeu et al., $2014^{23}$ & Yes & No & No & Yes & Yes & Yes & Yes & Yes & Yes & No & Yes & 8 \\
\hline Killane, et al., $2015^{24}$ & Yes & No & No & Yes & No & No & No & Yes & Yes & Yes & Yes & 6 \\
\hline Liao et al., $2015^{\mathrm{a} 25}$ & Yes & Yes & Yes & Yes & No & Yes & Yes & Yes & Yes & Yes & Yes & 10 \\
\hline Liao et al., $2015^{\mathrm{b} 26}$ & Yes & Yes & Yes & Yes & No & Yes & Yes & Yes & Yes & Yes & Yes & 10 \\
\hline Palacios-Navarro et al., $2015^{27}$ & Yes & No & No & Yes & No & No & No & Yes & Yes & No & Yes & 5 \\
\hline Ginis et al., $2016^{28}$ & Yes & Yes & No & Yes & Yes & No & No & Yes & Yes & Yes & Yes & 8 \\
\hline Strouwen et al., $2017^{29}$ & Yes & No & Yes & Yes & Yes & Yes & Yes & No & Yes & Yes & Yes & 9 \\
\hline Yang et al., $2016^{30}$ & Yes & Yes & Yes & Yes & No & No & No & Yes & Yes & Yes & Yes & 8 \\
\hline Gandolfi et al, $2017^{31}$ & Yes & Yes & No & Yes & No & Yes & Yes & Yes & Yes & Yes & Yes & 9 \\
\hline Ferraz et al., $2018^{32}$ & Yes & Yes & Yes & Yes & Yes & No & No & Yes & Yes & Yes & Yes & 9 \\
\hline Dantas et al., $2018^{33}$ & Yes & No & No & No & No & No & No & Yes & Yes & Yes & Yes & 5 \\
\hline Melo et al., $2018^{34}$ & Yes & Yes & Yes & Yes & Yes & Yes & Yes & Yes & Yes & Yes & Yes & 11 \\
\hline Alves et al., $2018^{35}$ & Yes & Yes & Yes & Yes & No & No & No & Yes & Yes & Yes & Yes & 8 \\
\hline Song et al., $2018^{36}$ & Yes & Yes & Yes & Yes & Yes & Yes & Yes & Yes & Yes & Yes & Yes & 11 \\
\hline
\end{tabular}

1. Eligibility criteria; 2. Random distribution of subjects in each group; 3 . Secret allocation of subjects; 4 . Similar groups regarding the most important prognosis; 5 . Blind participation of subjects; 6 . Blind participation of therapists; 7. Blind examiners; 8 . At least one key result obtained in more than $85 \%$ of subjects; 9 . Subjects received treatment or control condition; 10 . Intergroup statistical comparisons have been performed for at least one key outcome; 11 . Presence of precision and variability measures.

balance. The association of static and dynamic balance and gait speed improvement may explain the less severe PD motor symptoms after training, measured by the UPDRS-III motor score, as reported by Mirelman et al., 2011; $;{ }^{19}$ Killane et al., 2015; ${ }^{24}$ Liao et al., 2015; $; 5$ Yang et al., 2015; ${ }^{29}$ Gandolfi et al., 2017; ${ }^{31}$ Ferraz et al., 2018; ${ }^{32}$ de Melo et al., 2018. ${ }^{34}$

VR dual-task gait training involves not only motor, but also cognitive training. Executive function, evaluated by parts $A$ and $B$ of the Trail-Making Test, improved in patients with PD after the training (Mirelman et al., $2011^{19}$ ). Patients also reported improvement in PD symptoms (Sony et al., 2018) ${ }^{36}$ and in quality of life, assessed by the PDQ-39 (Pompeu et al., 2014; ${ }^{22}$ Liao et al., 2015;26 Yang et al., 2015; ${ }^{29}$ Ferraz et al., 2018; ${ }^{32}$ de Melo et al., 2018 $8^{34}$ ).

Some authors suggest that VR tasks can improve motor learning in rehabilitation because they activate mirror neurons. When patients with PD imitate actions, mirror neurons are stimulated, allowing improvements in balance, global mobility and functional abilities. In addition, VR improves attention as patients focus on specific demands of games by recruiting cognitive, motor, oculomotor, cerebellar and limbic loops. ${ }^{37,38}$

Patients with PD have difficulty performing dual- or multiple-tasks. ${ }^{5-7}$ This difficulty occurs because patients must focus on specific and accurate motor patterns. Thus, the premotor cortex is activated to compensate for basal ganglia damage and deficiency in dopamine production. ${ }^{38,39}$ Therefore, in dual-tasks, cortical resources process the motor and cognitive components in parallel. ${ }^{38-40}$ Patients with PD employ their cognitive reserves to perform gait even in single-tasks, and performance is seriously impacted in dual-tasks. ${ }^{41}$

Dual-task training should be part of the rehabilitation process of $\mathrm{PD}$ patients who have difficulty performing cognitive-motor tasks. ${ }^{42,43}$ Besides, patients in the initial stages of PD should perform dual-task training 
to prevent or delay these deficits. ${ }^{42}$ In a recent study, Fernandes et al. (2017) ${ }^{44}$ showed that the anticipatory postural adjustments during gait initiation were impaired in patients with PD. The authors reported an activation failure of the tibialis anterior muscle in both single- and dual-task conditions. Therefore, exercises that involve repeated tibialis anterior activation, such as step climbing, ${ }^{36}$ are important and should be included in rehabilitation programs for patients with PD. Song et al. (2018) combined VR with a step climbing task. ${ }^{36}$ Although patients reported mobility improvement, the authors failed to find a significant effect of VR step climbing training on TUG performance. Based on the study by Fernandes et al. (2017), an electromyographicbased analysis may be more sensitive for detecting postural control improvement than TUG, particularly in early-stage PD patients. ${ }^{44}$

The studies reviewed in the present study showed that VR dual-task training is effective, although the specific guidelines of dual-task protocols have not yet been defined. ${ }^{39}$ VR optimizes the benefits of dual-task training, such as task automation and more efficient task-related network integration. ${ }^{30}$ Visuomotor training can help the reorganization and maintenance of the normal circuitry that connects the motor cortex with the basal ganglia via the thalamus or cerebellum..$^{43}$ Visuomotor training can involve temporal or spatial stimuli, which regulate and facilitate repetitive movements by providing explicit targets. Visual cues have immediate effects on gait and many studies shown that effects were retained and associated with a higher quality of life in PD. $45-47$

The study by Esculier et al. $(2014)^{23}$ showed that lower limb corticomotor excitability increased during the observation, imagery and imitation of actions. Transcranial magnetic stimulation assessed motor evoked potentials in quadriceps femoris and soleus muscles before and after six weeks of training. The authors compared the effects of rehabilitation using Wii Fit ${ }^{\mathrm{TM}}$ in PD patients with controls. Although only eight people were included in each group, significant improvements in balance, gait speed and mobility were observed after a six-week training program. Increased cortical activity was observed in healthy individuals and in PD patients when they were learning new visuomotor tasks. Cortical activity decreased as learning progressed in healthy individuals, but patients with PD still needed to employ much attention even after several sessions of visuomotor training. ${ }^{20}$

VR dual-task gait training involves executive function, ${ }^{19}$ a predictor of balance deficits in patients with PD. ${ }^{48}$ VR tasks involve gait control and meet environmental demands (e.g. risk detection). Therefore, they can prevent falls and increase functional independence in patients with PD. VR dual-task gait training promotes ecological learning of selecting, planning and monitoring motor programs and of assessing cognitive resources (working memory and attention). These cognitive resources are affected by PD and frequently associated with falls risk and functional dependence. It is important to highlight the increase in motivation promoted by VR, which favours engagement and more efficient motor control and may explain the functional gains even in more severe patients.

Limitations of the present study included the fact that the variability in assessment and training protocols and in VR characteristics hindered meta-analysis. Evaluation follow-up times were also variable and only available in six (Espay et al., 2011;18 Mirelman et al., 2011; $;^{19}$ Liao et al., 2015; ${ }^{25}$ Ginis et al., 2016; ${ }^{28}$ Strouwen et al., 2017;30 Melo et al., 2018 ${ }^{34}$ ) of the 19 studies. New therapeutic strategies for patients with PD, such as VR dual-task gait training, involve integrative and low-cost approaches. Patients with PD must be considered within a biopsychosocial context, as they typically have to deal with the neurodegenerative disease for over 30 years of their life time. VR can be useful for dual-task gait training in patients with $\mathrm{PD}$, providing higher engagement and motivation.

In conclusion, VR dual-task gait training promotes gait improvement in patients with PD. Further studies should evaluate and compare the effects of specific rehabilitation programs in order to provide standardized guidelines for dual-task gait training in PD.

Author contributions. All authors contributed significantly to, and approved, the content of this manuscript.

\section{REFERENCES}

1. Koerts J, Tucha L, Lange K, Tucha O. The influence of cognitive reserve on cognition in Parkinson's disease. J Neural Transm. 2013;120:593-6.

2. Springer S, Giladin, Peretz C, Yogev G, Simon E, Hausdorff JM. Dualtasking effects on gait variability: The role of aging, falls, and executive function. Mov Disord. 2006;21(7):950-7.
3. Souza CO, Voos MC, Barbosa AF, Chen J, Francato DCV, Milosevic M, et al. Relationship between posturography, clinical balance and executive function in Parkinson's disease. J Mot Behav. 2019;51(2):212-21.

4. Rochester L, Burn DJ, Woods G, Godwin J, Nieuwboer A. Does auditory rhythmical cueing improve gait in people with Parkinson's disease 
and cognitive impairment? UH feasibility study. Mov Disord. 2009;24(6): 839-45.

5. Fernandes A, Coelho T, Vitoria A, Ferreira A, Santos R, Rocha N, et al. Standing balance in individuals with Parkinson's disease during single and dual-task conditions. Gait Posture. 2015;42(3):323-8.

6. Souza ASP, Silva A, Tavares JMRS. Biomechanical and neurophysiological mechanisms related to postural control and efficiency of movement: a review. Somatosens Mot Res. 2012;29(4):131-43.

7. Fernandes A, Souza ASP, Couras J, Rocha N, Tavares JMRS. Influence of dual-task on sit-to-stand-to-sit postural control in Parkinson's disease. Med Eng Phys. 2015;37:1070-5.

8. Brauer SG, Woollacott MH, Lamont R, Clewett S, O'Sullivan J, Silburn $\mathrm{P}$, et al. Single and dual task gait training in people with Parkinson's Disease: a protocol for a randomised controlled trial. BMC Neurology. 2011;11:90.

9. Van Diest M, Lamoth CJC, Stegenga J, Verkerke GJ and Postema K. Exergaming for balance training of elderly: state of the art and future developments. J Neuroeng Rehabil. 2013;10:101

10. Mirelman A, Maidan I, Deutsch JE. Virtual reality and motor imagery: Promising tools for assessment and therapy in Parkinson's disease. Mov Disord. 2013;28(11):1597-608.

11. Shumway-Cook A, Brauer S, Woollacott M. Predicting the probability for falls in community-dwelling older adults using the Timed Up \& Go Test. Phys Ther. 2000;80(9):896-903.

12. Burdea GC. Virtual rehabilitation-benefits and challenges. Methods Inf Med 2003;42(5):519-23.

13. Keshner EA. Virtual reality and physical rehabilitation: a new toy or a new research and rehabilitation tool? J Neuroeng Rehabil. 2004;1(1):8.

14. Barry G, Galna B, Rochester $L$. The role of exergaming in Parkinson's disease rehabilitation: a systematic review of the evidence. J Neuroeng Rehabil. 2014;7(11):33.

15. Dockx K, Bekkers EMJ, Van den Bergh V, Ginis P, Rochester L, Hausdorff $\mathrm{JM}$, et al. Virtual reality for rehabilitation in Parkinson's disease (Review). Cochrane Database of Systematic Reviews. 2016;12:CD010760.

16. Vieira GP, Araujo DFGH, Leite MAA, Orsini M, Correa CL. Virtual reality in physical rehabilitation of patients with Parkinson's disease. J Hum Growth Dev 2014;24(1):31-41.

17. Verhagen AP, de Vet HC, de Bie RA, Kessels AG, Boers M, Bouter LM, et al. The Delphi list: a criteria list for quality assessment of randomized clinical trials for conducting systematic reviews developed by Delphi list: a criteria list for quality assessment of randomized clinical trials for conducting systematic reviews developed by Delph consensus. J Clin Epidemiol. 1998:51(12):1235-41.

18. Espay AJ, Baram Y, Dwivedi AK, Shukla R, Gartner M, Gaines L, et al. At-home training with closed-loop augmented-reality cueing device for improving gait in patients with Parkinson disease. J Rehabil Res Dev. 2010;47(6):573-81.

19. Mirelman A, Maidan I, Herman T, Deutsch JE, Giladi N, Hausdorff JM Virtual Reality for Gait Training: Can it Induce Motor Learning to Enhance Complex Walking and Reduce Fall Risk in Patients with Parkinson's Disease? Phys Ther. 2011;80(9):896-903.

20. Esculier, JF, Vaudrin, J, Tremblay, LE. Corticomotor Excitability in Parkinson's Disease During Observation, Imagery and Imitation of Action: Effects of Rehabilitation Using Wii Fit and Comparison to Healthy Controls. J Parkinsons Disease. 2014;4:67-75.

21. Mhatre PV, Vilares I, Stibb SM, Albert MV, Pickering L, lak CMM, et al. Wii Fit balance board playing improves balance and gait in Parkinson disease. PM R. 2013;5(9):769-77.

22. Pompeu JE, Arduini LA, Botelho AR, Fonseca MB, Pompeu SM, TorrianiPasin C, Deutsch JE. Feasibility, safety and outcomes of playing Kinect Adventures! for people with Parkinson's disease: a pilot study. Physiotherapy. 2014;100(2):162-8.

23. Esculier JF, Vaudrin J, Bériault P, Gagnon K and tremblay L. HomeBased balance training programme using Wii Fit with balance board for parkinson's disease: A pilot study. J Rehabil Med. 2012;44:144-50.

24. Killane I, Fearon C, Newman L, McDonnell C, Waechter SM, Sons K, Lynch T, Reilly RB. Dual motor-cognitive virtual reality training impacts dual-task performance in freezing of gait. IEEE J Biomed Health Inform. 2015;19(6):1855-61

25. Liao $Y Y$, Yang YR, Wu YR, Wang RY. VR-based Wii fit training in improving muscle strength, sensory integration ability and walking abilities in patients with PD. Int J Gerontol. 2015;9(4):190-5.

26. Liao YY, Yang YR, Cheng SJ, Wu YR, Fuh JL, Wang RY. VR-based training to improve obstacle-crossing performance and dynamic balance in patients with PD. Neurorehabil Neural Repair. 2015;29(7):658-67.

27. Palacios-Navarro PG, Magariño Gl, Lorente RP. A kinect-based system for lower limb rehabilitation in Parkinson's disease patients: a pilot study. J Med Syst. 2015;39(9):103.
28. Ginis P, Nieuwboer A, Dorfman M, Ferrari A, Gazit E, Canning CG, Rocchi L, Chiari L, Hausdorff JM, Mirelman A. Feasibility and effects of home-base smartphone-delivered automated feedback training for gait in people with Parkinson's disease: A pilot randomized controlled trial. Parkins Related Disord. 2016;22:28-34.

29. Yang WC, Wang HK, Wu RM, Lo CS, Lin KH. Home-based virtual reality balance training and conventional balance training in Parkinson's disease: A randomized controlled trial. J Formos Med Assoc. 2016; 115(9):734-43.

30. Strouwen C, Molenaar EALM, Münks L, Keus SHJ, Zijlmans CM, Vandenberghe W, Bloem BR, Nieuwboer A. Training dual tasks together or apart in Parkinson's disease: results from the duality trial. Mov Disord. 2017;32(8):1201-10.

31. Gandolfi M, Geroin C, Dimitrova E, Boldrini P, Waldner A, Bonadiman S, Picelli A, Regazzo S, Stirbu E, Primon D, Bosello C, Gravina AR, Peron L, Trevisan M, Garcia AC, Menel A, Bloccari L, Valè N, Saltuari L, Tinazzi M, Smania N. Virtual reality telerehabilitation for postural instability in Parkinson's disease: a multicenter, single-blind, randomized, controlled trial. Biomed Res Int. 2017:7962826.

32. Ferraz DD, Trippo KV, Duarte GP, Neto MG, Bernardes SKO, Filho JO. The effects of functional training, bicycle exercise, and exergaming on walking capacity of elderly patients with Parkinson disease: a pilot randomized controlled single-blinded trial. Arch Phys Med Rehabil. 2018; 99(5):826-33

33. Dantas IV, Leal JC, Hilgert LS, Allegretti ALC, Mendes FAS. Training healthy persons and individuals with Parkinson's disease to use Xbox Kinect games: a preliminary study. Int J Ther Rehab. 2018;25(6).

34. de Melo GEL, Kleiner AFR, Lopes JBP, Dumont AJL, Lazzari RD, Gall $\mathrm{M}$, Oliveira CS. Effect of virtual reality training on walking distance and physical fitness in individuals with Parkinson's disease. NeuroRehabilitation. 2018;42(4):473-80.

35. Alves MLM, Mesquita BS, Morais WS, Leal JC, Satler CE, Dos Santos Mendes FA. Nintendo Wii versus Xbox Kinect for assisting people with Parkinson's disease. Percept Mot Skills. 2018;125(3):546-65.

36. Song J, Paul SS, Caetano MJD, Smith S, Dibble LE, Love R, et al. Home-based step training using videogame technology in people with Parkinson's disease: a single-blinded randomised controlled trial. Clin Rehabil. 2018:32(3):299-311.

37. Mirelman A, Rochester L, Reelick M, Nieuwhof F, Pelosin E, Abbruzzese G, Dockx K, et al. V-TIME: a treadmill training program augmented by virtual reality to decrease fall risk in older adults: study design of a randomized controlled trial. BMC Neurology. 2013;13:15.

38. Fernandes Â, Rocha N, Santos R \& Manuel JRST. Effects of dual task training on balance and executive functions in Parkinson's disease: $A$ pilot study. Somatosens Mot Res. 2015;32(2):122-7.

39. Wu T, Hallett M. Dual task interference in Parkinson's disease. Touch Briefings 2009;34-7.

40. Brauer SG, Morris ME. Can people with Parkinson's disease improve dual tasking when walking? Gait Posture. 2010;31:229-33.

41. Fuller RL, Van Winkle EP, Anderson KE, Gruber-Baldini AL, Hill T, Zampierl C, Weiner WJ, Shulman LM. Corticomotor Excitability in Parkinson's Disease During Observation, Imagery and Imitation of Action: Effects of Rehabilitation Using Wii Fit and Comparison to Healthy Controls. $J$ Parkinsons Dis. 2014:4:67-75.

42. Fernandes A, Rocha N, Santos R, Tavares JMRS. Effects of dual-task training on balance and executive functions in Parkinson's disease: A pilot study, Somatosens Mot Res. 2015;32(2):122-7.

43. Fernandes A, Souza ASP, Rocha N, Tavares JMRS. Parkinson's disease and cognitive-motor dual-task: is motor prioritization possible in the early stages of the disease? J Mot Behav. 2016:48(4):377-83.

44. Fernandes A, Souza ASP, Rocha N, Tavares JMRS. The influence of a cognitive task on the postural phase of gait initiation in Parkinson's disease: an electromyographic-based analysis. Motor Control. 2017; 21(3):249-64.

45. Mak M, Hallett M. Effect of cued training on motor evoked potential and cortical silent period in people with Parkinson's disease. Clin Neurophysiol. 2013;124:545-50.

46. Nieuwboer A, Kwakkel G, Rochester L, Jones D, Van WE, Willems AM, et al. Cueing training in the home improves gait-related mobility in Parkinson's disease: the rescue trial. J Neurol Neurosurg Psychiatry. 2007;78:134e140.

47. Rochester L, Baker K, Hetherington V, Jones D, Willems AM, Kwakkel $G$, et al. Evidence for motor learning in Parkinson's disease: acquisition, automaticity and retention of cued gait performance after training with external rhythmical cues. Brain Res. 2010;1319:103e111.

48. Fernandes A, Mendes A, Rocha N, Tavares JMRS. Cognitive predictors of balance in Parkinson's disease. Somatosens Mot Res. 2016; 33(2):67-71. 